\title{
Ordering Characteristics of Anti-Heat Shock Protein 70 Antibody in Australasia
}

\author{
Adrian Yong Sing Lee 1,2,3 $^{1,3}$ \\ ${ }^{1}$ Department of Immunology, Westmead Hospital, Westmead, NSW, Australia \\ ${ }^{2}$ Department of Immunopathology, Institute of Clinical Pathology \& Medical Research, NSW Health Pathology, Westmead, \\ NSW, Australia \\ ${ }^{3}$ Westmead Clinical School, University of Sydney, Westmead, NSW, Australia
}

Dear Editor,

Anti-heat shock protein 70 (HSP70) IgG antibodies are commonly ordered serum autoantibodies in the investigation of sensorineural hearing loss (SNHL), either primary or associated with other conditions such as Meniere's disease. It is a biomarker for autoimmune SNHL that may respond to steroids; yet, lacks diagnostic sensitivity and specificity [1]. It may be found in around a quarter of patients with autoimmune SNHL and a third of patients with Meniére's disease [2].

The Westmead Immunology laboratory (Westmead Hospital) is a quaternary referral laboratory that performs testing of these autoantibodies in Australasia. We reviewed the ordering characteristics of anti-HSP70 over a 5-year period from 2016-2021. Testing was performed using a qualitative line immunoblot (ImmcoStripe, Trinity Biotech, Buffalo, NY, USA) as per manufacturer's instructions, and graded as not detected (negative), detected (positive) or equivocal for antiHSP70 IgG. Two experienced scientists independently performed the evaluation.

There were 856 tests for anti-HSP70 over the 5-year period with $28(3.3 \%)$ and $53(6.2 \%)$ specimens were equivocal and positive for the autoantibody, respectively. The mean age was

Received September 23, 2021 / Revised September 30, 2021

Accepted October 7, 2021

\section{Address for correspondence}

Adrian Yong Sing Lee, MB, BS

Department of Immunology, Westmead Hospital, Level 2 ICPMR,

Hawkesbury Road, Westmead NSW 2145, Australia

Tel +61-2-8890-6933/ Fax +61-2-9891-3889

E-mail adrian.lee1@health.nsw.gov.au

This is an Open Access article distributed under the terms of the Creative Commons Attribution Non-Commercial License (https://creativecommons.org/licenses/by-nc/4.0/) which permits unrestricted non-commercial use, distribution, and reproduction in any medium, provided the original work is properly cited.
$48.5 \pm 19.2$ years and the cohort consisted of 507 (59.2\%) females. As indicated by Table 1, there were no significant differences in these statistics according to the anti-HSP70 result.

The most common ordering specialties were otolaryngology $(576,67.3 \%)$, immunology $(88,10.3 \%)$, general practice $(71$, $8.3 \%)$, neurology $(67,7.8 \%)$ and rheumatology $(11,1.3 \%)$. Out of these specialties, otolaryngology patients had a reduced proportion of patients with a positive anti-HSP70 compared to their general cohort, perhaps reflecting the large proportion of patients with SNHL they evaluate (Table 1). Immunology referred a significantly higher proportion of patients with positive anti-HSP70 likely due to their involvement with patients with autoimmune SNHL (Table 1).

The clinical notes and medical records were reviewed for each request episode. Out of the 856 episodes, these were available for 672 episodes (78.5\%). The most common reason for requesting the autoantibody were for the investigation of hearing loss (453/672, 67.4\%). The frequency of negative, equivo$\mathrm{cal}$, and positive anti-HSP70 antibodies did not differ according to the main reason for requesting the test (Table 1).

The surprising lack of correlations with the presence of anti-HSP70 in this cohort may relate to the heterogeneous state and aetiology of patients with SNHL since the autoantibody tends to be present in patients with active disease over inactive disease [3]. Moreover, some of these patients may have been on treatment which was not possible to ascertain from clinical notes alone. Other studies have maintained that antiHSP70 is of limited clinical utility in screening patients with deafness [4], and controversies exist about the assay used to measure these autoantibodies [5].

This is the first real-world review of the performance of anti-HSP70 in a general laboratory population. In this study cohort, age, sex, and clinical reason for requesting the test 
Table 1. Demographic details, requesting specialty and clinical reasons for requesting anti-heat shock protein 70 (HSP70) antibody

\begin{tabular}{|c|c|c|c|c|}
\hline & Not detected $(n=775)$ & Equivocal $(n=28)$ & Detected $(n=53)$ & p-value \\
\hline Female & $460(59.4)$ & $14(50.0)$ & $33(62.3)$ & 0.550 \\
\hline Age (yr) & $48.4 \pm 19.2$ & $51.1 \pm 17.7$ & $48.2 \pm 19.6$ & 0.761 \\
\hline \multicolumn{5}{|l|}{ Specialty } \\
\hline Otolaryngology & $531(68.5)$ & $19(67.9)$ & $26(49.1)$ & 0.014 \\
\hline Immunology & $75(9.7)$ & $2(7.1)$ & $11(20.8)$ & 0.034 \\
\hline General practice & $63(8.1)$ & $1(3.6)$ & 7 (13.2) & 0.282 \\
\hline Neurology & $56(7.2)$ & $3(10.7)$ & $8(15.1)$ & 0.101 \\
\hline Rheumatology & $10(1.3)$ & $0(0.0)$ & $1(1.9)$ & 0.573 \\
\hline \multicolumn{5}{|l|}{ Clinical reason* } \\
\hline Total $^{\dagger}$ & 606 & 25 & 41 & \\
\hline SNHL & $275(45.3)$ & $11(44.4)$ & $11(26.8)$ & 0.069 \\
\hline Hearing loss & $135(22.2)$ & $6(24.0)$ & $15(36.6)$ & 0.110 \\
\hline Meniere's & $10(1.7)$ & $0(0.0)$ & $1(2.4)$ & 0.672 \\
\hline Tinnitus & $10(1.7)$ & $1(4.0)$ & $0(0.0)$ & 0.654 \\
\hline Dizziness & $7(1.2)$ & $0(0.0)$ & $2(4.9)$ & 0.093 \\
\hline
\end{tabular}

Data are presented as $\mathrm{n}$ (\%) or mean \pm standard deviation. Stratification according anti-HSP70 is provided as well as $p$-values for any significant differences. *the remaining cases are a collection of miscellaneous reasons; ${ }^{\dagger}$ requests without clinical notes were excluded from analysis. When the medical/clinical notes did not specify what type of hearing loss that patient had (i.e., conductive vs. sensorineural [SNHL] vs. mixed), the general "hearing loss" label was used. Those patients that had a confirmed SNHL were isolated out as "SNHL" patients. SNHL, sensorineural hearing loss

were not helpful variables in predicting the positivity of antiHSP70. However, future studies would be helpful in ascertaining the other predictors for patients that present with steroidresponsive SNHL.

\section{Acknowledgments}

The author wishes to thank the staff of the Immunopathology Laboratory for their technical expertise.

\section{Conflicts of interest}

The author has no financial conflicts of interest.

\section{ORCID iD}

Adrian Yong Sing Lee https://orcid.org/0000-0002-5179-4803

\section{REFERENCES}

1) Bonaguri C, Orsoni JG, Zavota L, Monica C, Russo A, Pellistri I, et al. Anti- $68 \mathrm{kDa}$ antibodies in autoimmune sensorineural hearing loss: are these autoantibodies really a diagnostic tool? Autoimmunity 2007;40:73-8.

2) Gottschlich S, Billings PB, Keithley EM, Weisman MH, Harris JP. Assessment of serum antibodies in patients with rapidly progressive sensorineural hearing loss and Menière's disease. Laryngoscope 1995;105:1347-52.

3) Moscicki RA, San Martin JE, Quintero CH, Rauch SD, Nadol JB Jr, Bloch KJ. Serum antibody to inner ear proteins in patients with progressive hearing loss. Correlation with disease activity and response to corticosteroid treatment. JAMA 1994;272:611-6.

4) Samuelsson AK, Hydén D, Roberg M, Skogh T. Evaluation of antihsp70 antibody screening in sudden deafness. Ear Hear 2003;24: 233-5.

5) Tebo AE, Jaskowski TD, Keith LL, Litwin CM, Hill HR. Comparison of immune assays for the detection of anti-HSP70 antibodies in patients with idiopathic sensorineural hearing loss. Clin Chim Acta 2007;381:140-4. 